\title{
Influence of Stromal Components on Lung Cancer Carcinogenesis
}

\author{
Laurie A. Shuman Moss* and William G. Stetler-Stevenson* \\ Radiation Oncology Branch, National Cancer Institute, NIH, Bethesda, MD 20892-1500, USA
}

\begin{abstract}
The association between tumor growth and angiogenesis was first observed over 100 years ago. Since then, research has shown the dependence of tumor growth on angiogenesis and the ability of cancer cells to alter the stromal microenvironment. Technological advancements have enabled researchers to identify cell types within a tumor, identify chemokines, cytokines, and growth factors secreted by tumor cells, show the interaction between tumor cells and stroma, and investigate the function of distinct genes using knockout and transgenic mouse technology. This review provides an overview of tumor growth, emphasizing research using in vivo mouse models on vascular endothelial growth factor (VEGF), fibrinogen, fibronectin, plasminogen, and MMPs in primary tumor growth and metastasis of lung cancer in particular.
\end{abstract}

Keywords: Lung cancer; VEGF, Fibronectin; Fibrinogen; Plasminogen; Matrix metalloproteinases; TIMPs

\section{Introduction}

Many years ago it was observed that growing tumors formed new blood vessels, found mainly at the periphery of infiltrating tumors and that sarcoma and carcinoma cells were able to invade blood vessels [1,2]. Work continued in this area and in 1971 Folkman proposed that blocking angiogenesis could inhibit tumor growth $[3,4]$. Concurrently, research on the components within the tumor stroma revealed the presence of fibrinogen or fibrin implying that tumor cells were altering the surrounding stroma for growth and expansion [5]. Tissue architecture is normally maintained via cell-cell and cell-extracellular matrix cross-talk. Malignant cells do not abide by the signals that maintain normal tissue architecture. Molecular mechanisms observed in processes of physiological invasion, including neovascularization, wound healing, and neurite outgrowth, as well as embryogenesis, are also seen in malignant invasion. In 1986 Dvorak said that tumor cell growth and biology is "wound healing gone awry," where tumor stroma formation parallels host wound healing processes [2]. Tumor cells secrete Vascular Endothelial Growth Factor (VEGF), also called Vascular Permeability Factor (VPF), leading to increased vascular permeability and angiogenesis. VEGF causes endothelial cell proliferation, separation, migration, and survival. VEGF expression is stimulated by a variety of conditions common to many tumors, including hypoxia and low $\mathrm{pH}$, and by various genetic mutations, such as $K$-ras, $p 53$, and HER2/ErbB2 [6,7].

It was once thought that tumor cells could not synthesize fibrinogen or fibrin and that the presence of tumor fibrin was due to extravasation and extravascular clotting of plasma fibrinogen. However, recent data show that A549 lung cancer cells, as well as DU-145 and PC-3 prostate cancer cells, synthesize fibrinogen which enhances FGF-2 stimulated tumor growth of these cells [8]. Radioactive labeled fibrinogen has been shown to enter the tumor microenvironment five times faster than control tissue due to leakage of the vasculature. Fibrinogen influx into tumors happens continuously and at a consistent rate amongst many different tumor types. The difference in fibrin deposition within tumors is related to the amount of plasminogen activators secreted by the tumor cells and this affects fibrinolysis $[2,5,9]$.

The continuous VEGF secretion and fibrin deposition signals new blood vessel growth, the recruitment of fibroblasts and macrophages, as well as, collagen deposition leading to angiogenesis and continued tumor growth. Both type III collagen and type I collagen are found within the tumor stroma. Type III collagen is found in less mature tumor stroma and type I collagen is found in older sclerotic tumor tissue $[2,10,11]$.

In the past decade, Sivridis and colleagues have characterized the process of stromatogenesis, or formation of new stroma at sites of active tumor invasion, in many different tumor types isolated from patients. The antigenic phenotype of stromal cells within the newly formed stroma at the invading tumor front has increased expression of a-Smooth Muscle Actin (SMA), Thymidine Phosphorylase (TP), Secreted Protein Acidic and Rich in Cysteine (SPARC), and a higher MIB-1 proliferation index, while vimentin and intestinal-type alkaline phosphatase (IAP) expression is decreased. The extracellular matrix also displays a similar phenotype of stromatogenesis at the invading tumor front with increased expression of SPARC, CD31 as a marker of angiogenesis, and type III collagen, and decreased expression of type-I collagen and fibronectin. The stroma found in the center of a tumor mass is more dense to support tumor growth, in contrast to the loose network of stromal cells and extracellular matrix formed during stromatogenesis. Tumor cells can more easily penetrate this newly formed stroma, enhancing invasion [10-12]. Cekanova et al. showed that proliferation of two human pulmonary adenocarcinoma cell lines, NCI-H322 and A549, was increased when co-culture with fibroblasts isolated from a healthy patient, CCD-19Lu. Proliferation of both adenocarcinoma cell lines was even more pronounced when cocultured with HLF-A fibroblasts that were isolated from a patient with epidermoid carcinoma of the lung. The enhanced proliferation was attributed to amphiregulin and transforming growth factor- $\alpha$ released by the fibroblasts [13].

Local tissue invasion and subsequent dissemination of tumor

*Corresponding author: Laurie Shuman Moss, Ph.D. or William G. Stetler Stevenson, M.D., Ph.D., Radiation Oncology Branch, National Cancer Institute NIH, BIdg 10, Room 2A33, 10 Center Dr, MSC 1500, Bethesda, MD 20892-1500, USA, E-mail: shumanmossla@mail.nih.gov, sstevenw@mail.nih.gov

Received April 09, 2013; Accepted June 15, 2013; Published June 25, 2013

Citation: Moss LAS, Stevenson WGS (2013) Influence of Stromal Components on Lung Cancer Carcinogenesis. J Carcinogene Mutagene S13: 008. doi:10.4172/2157-2518.S13-008

Copyright: (C) 2013 Moss LAS, et al. This is an open-access article distributed under the terms of the Creative Commons Attribution License, which permits unrestricted use, distribution, and reproduction in any medium, provided the original author and source are credited. 
cells leads to life-threatening metastases. During tumor cell invasion, four distinct processes occur in concert; expression of cell surface molecules, including integrins and cadherins, are altered to reduce cell adhesion; tumor cell motility; as well as ECM remodeling, is increased; and angiogenesis all occur [10]. Matrix metalloproteinases (MMPs) are upregulated in many types of tumors. High expression of MMPs in serum or tumor samples correlates with poor prognosis [14]. MMPs are zinc-dependent endopeptidases classified by molecular structure as secreted or membrane-bound and are initially synthesized as inactive proenzymes and later activated via the "cysteine switch" mechanism where the pro-peptide domain is cleaved. MMPs are secreted by many cell types, including fibroblasts, macrophages, neutrophils, and tumor cells. MMP activity is regulated by a family of proteins called the Tissue Inhibitors of Metalloproteinases (TIMPS) of which there are four members; TIMP-1, TIMP-2, TIMP-3, and TIMP-4. The TIMP proteins function to block MMP-mediated proteolysis by noncovalently binding to the MMP active site in a 1:1 stoichiometric ratio [14-17].

Technological advancements have enabled sequencing of tumor DNA samples to identify novel genes for prognosis or therapeutics. In addition, the ability to generate murine knockout and transgenic models allows identification of gene function in tumor growth. This review will focus on various in vivo mouse models that have been established to examine distinct roles for VEGF, fibrinogen, fibronectin, plasminogen, and MMPs in primary tumor growth and metastasis of lung cancer.

\section{VEGF in Lung Cancer}

Mammalian cells are located within 100-200 $\mu$ m of blood vessels due to the need for oxygen and nutrients for survival [18]. For this reason tumor cells require blood vessels in order to grow beyond a few millimeters in size and disseminate to secondary sites. This happens when the balance between pro- and anti-angiogenic factors no longer exists due to increased pro-angiogenic mechanisms, thus flipping the "angiogenic switch" on [19,20]. VEGF is a potent angiogenic factor found overexpressed in many solid tumors and high serum levels of VEGF have been shown to correlate with poor prognosis in a variety of cancers. VEGF expression is stimulated in hypoxic conditions and has been shown to induce proliferation, sprouting, migration, and enhance survival of endothelial cells. In addition, increased VEGF production recruits inflammatory cells to the area and aids in increased expression of proteases from endothelial cells resulting in pericellular matrix degradation necessary for angiogenesis [19-21].

Homo- and heterozygous VEGF knockout mice are embryonic lethal due to defects in vascular development [22]. Therefore, studies using conditional knockdown of VEGF expression in tissues or tumor cells or blocking VEGF receptors have shown the role VEGF plays in primary tumor growth and metastasis. Ma and colleagues used shRNA targeting VEGF in A549 lung adenocarcinoma cells to show that decreased VEGF expression in tumor cells resulted in decreased tumor growth and results were more profound when the chemotherapy agent, cis-Diclorodiamminoplatino (DDP), was administered concomitantly [23]. The VEGF pathway is the focus of many treatment strategies currently in preclinical and clinical development. Bevacizumab is an anti-VEGF antibody approved by the US Food and Drug Administration for cancer treatment. Bevacizumab can be used alone or in combination to treat glioblastoma, colorectal, non-small cell lung, and renal cell cancers. In addition to targeting VEGF directly, other therapeutic targets include EGFR, HER-2, COX-2, and HIF-1a, which can indirectly regulate the VEGF pathway decreasing angiogenesis $[21,24,25]$.
Alternative splicing of the VEGF gene gives rise to different isoforms, including VEGF-121, 165, 189, and 206. These isoforms differ in their biochemical and biological functions in angiogenesis and their role in tumor progression is being elucidated. $\mathrm{VEFG}_{121}$ has the highest bioavailability of the VEGF isoforms because it is freely released from the cell and does not bind heparin. The expression level of VEGF ${ }_{121}$ mRNA has been implicated as a prognostic indicator of early stage lung cancer. High levels of VEGF ${ }_{121}$ mRNA were found to be associated with metastasis to the lymph nodes and shorter patient survival of Non-Small Cell Lung Cancer (NSCLC) [21,26]. Additionally, Guo and colleagues reported that $\mathrm{VEGF}_{121}$ mRNA levels correlate with the aggressiveness and F-18 fluorodeoxyglucose (FDG) uptake, a glucose analogue used to measure tumor metabolism by positron emission tomography (PET), in patients with lung adenocarcinoma [26]. Yuan et al. overexpressed VEGF-121, 165, and 189 isoforms in the human lung adenocarcinoma cell line, CL1-0, which has low tumorigenic and metastatic potential and low levels of endogenous VEGF. The number, size, and location of blood vessels differed in the tumors generated from the three VEGF isoforms. The $\mathrm{VEGF}_{121}$-overexpressing tumors had the lowest-density microvessels located mainly at the tumor rim, while the $\mathrm{VEGF}_{189}$ overexpressing tumors had the smallest, most-dense microvessels that penetrated into the center of the tumor [27]. These studies reiterate both the complexity of and expanding research in the role VEGF plays in lung cancer progression.

\section{Fibrinogen in Lung Cancer}

Fibrin(ogen) preserves vascular integrity and homeostasis via coagulant and fibrinolysis cascades. Fibrinogen helps form fibrin clots and a fibrin matrix of crosslinked fibrin monomers that acts as a scaffold for inflammatory cells and platelets. Malignant human and animal tumor cells overexpress procoagulant and fibrinolytic factors, including tissue factor, plasminogen activator, plasminogen receptor, plasminogen inhibitor, and fibrinogen receptors and binding proteins. Disruption of the fibrinogen $\mathrm{A} \alpha$ chain gene ablates all fibrinogen polypeptides from circulation and mice lacking the fibrinogen Aa chain gene show a decrease in both experimental (i.v. injection) and spontaneous (s.c. injection) metastasis of Lewis lung carcinoma cells $[28,29]$. Fibrinogen deficiency is particularly important to circulating tumor cells, as fibrinogen deficiency did not affect tumor stroma formation or primary tumor growth. Mice lacking natural killer cells (NK) were crossed with fibrinogen deficient mice to generate mice lacking both NK and fibrinogen function. In the absence of NK cell function, formation of metastatic foci after i.v. injection of Lewis lung carcinoma cells remained the same independent of fibrinogen expression suggesting that the decrease in metastatic foci in fibrinogen deficient mice is related to natural killer cell elimination of tumor cells [30]. These studies emphasize the role of host generated versus tumor cell generated fibronectin in stages of tumor progression and potential mechanism(s) for lung colonization.

\section{Fibronectin in Lung Cancer}

Plasma fibronectin is an abundant adhesion protein found in blood and clotted plasma. Plasma fibronectin is incorporated into fibrin clots and becomes covalently linked to fibrin and can bind members of the integrin family of receptors, including integrin $\alpha_{\mathrm{v}} \beta_{3}$. Tumors express a variety of integrin receptors, however, tumors expressing activated $\alpha_{v} \beta_{3}$ metastasize aggressively and metastasis can be inhibited with $\alpha_{v} \beta_{3}$ antibodies, RGD-containing peptides, or reducing expression via shRNA targeting $\alpha_{v} \beta_{3}$ [31]. In addition, expression of fibronectin in malignant tumors correlates with an invasive and metastatic phenotype. 
Fibronectin knockout mice are embryonic lethal, therefore, fibronectin deficient mice generated using the CRE-lox system to postnatally delete the hepatic fibronectin expression have been useful to study the role of plasma fibronectin in tumor growth and metastasis. Plasma fibronectin deficient mice show decreased lung metastasis of Lewis lung carcinoma and B16F1 melanoma cells [31,32]. Jia et al., generated a spontaneous metastatic model of human lung cancer, SPC-A-1sci. These cells have increased expression of fibronectin and vimentin and low expression of ZO-1 and E-cadherin indicative of cells that have gone through an epithelial to mesenchymal transition (EMT). Inhibiting fibronectin expression via shRNA in SPC-A-1sci cells reduced metastasis to the lung [33].

\section{Plasminogen in Lung Cancer}

Plasmin is a serine protease formed from the cleavage of plasminogen by urokinase-type (uPA) or tissue-type (tPA) plasminogen activators. Plasmin has $10^{4}-10^{6}$ fold higher activity than plasminogen and tumor cells show increased expression of components of the uPA system. Plasminogen activators released from tumor cells enhance the conversion of plasminogen to plasmin thus catalyzing extracellular matrix and basement membrane degradation facilitating cancer cell invasion and cancer-cell directed tissue remodeling. Plasminogen is not essential in development since plasminogen knockout mice develop to term, grow to adulthood, and are fertile [34]. Plasminogen knockout mice, along with uPA and tPA knockout mice develop intra- and extra-vascular fibrin deposits leading to widespread organ damage, low body weight, and a short life span. This affect was more pronounced in plasminogen knockout mice implying that uPA can substitute for tPA in fibrinolysis $[35,36]$. In addition, plasminogen knockout mice showed decreased recruitment of leukocytes to inflammatory sites. Transplanted Lewis lung carcinoma exhibited delayed growth and fewer regional lymph node metastases in plasminogen knockout mice, but the number of metastatic foci in the lungs remained the same [37]. Recently, Casar and colleagues reported that plasmin is necessary for the cleavage of the cell surface CUB domain-containing protein-1 (CDCP1) during early stages of lung colonization. High expression of CDCP1 has been found in a variety of cancers, including colon, breast, stomach, prostate, kidney, lung, and pancreas. Casar et al. overexpressed CDCP1 in human embryonic kidney cells, HEK293, that have no detectable levels of CDCP1 expression. CDCP1 expression increased lung colonization of these cells. Lung retention of HEK293 overexpressing CDCP1 was significantly reduced in plasminogen knockout mice, along with Akt activation, while PARP1 cleavage was increased supporting the idea that cleavage of CDCP1 by plasmin promotes cancer cell survival by activating the Akt pathway to suppress PARP1-induced apoptosis of cancer cells [38].

\section{Matrix Metalloproteinase (MMP) Activity in Lung Cancer}

MMPs have been shown to be upregulated in almost all human and animal tumors and cell lines [39]. The stage of tumor progression is positively correlated with the expression of MMP-1, 2,3,7,9,11, and 14 [40]. Increased MMP expression in tumor cells may disrupt tissue homeostasis and facilitate invasion of surrounding tissue and metastasis. Expression of distinct MMP family members has been associated with particular tumors and metastatic sites. For example, MMP-14, also referred to as membrane-type-MMP (MT1-MMP), has been detected in $80 \%$ of brain metastases arising from lung adenocarcinoma, while increased MMP-9 expression is frequently found in liver metastases from colorectal cancer [41,42]. The use of MMP-2, or gelatinase A, deficient mice show decreased primary tumor growth, angiogenesis, and metastasis of B16-BL6 melanoma cells and Lewis lung carcinoma [43]. In addition, MMP-9, or gelatinase B, deficient mice also showed decreased metastasis of B16-BL6 and Lewis lung carcinoma in an experimental metastasis model [44]. Rather than blocking expression of MMPs in the host, much research focuses on overexpressing or reducing expression of MMPs in tumor cells. Adenovirus-mediated inhibition of uPAR and MMP-9 decreased primary tumor growth and metastasis of A549 lung cancer cells, whereas overexpression of MMP14 in prostate cancer cells enhanced metastasis to the lung $[45,46]$.

Some challenges exist in determining the exact role each MMP has in tumor progression. MMPs share substrate specificity and other MMP family members can be upregulated to compensate for reduced expression of another family member. In addition, the expression of MMPs at metastatic sites could be attributed to the dissemination of the more invasive tumor cells in the primary tumor population or the establishment of a pre-metastatic niche. Hiratsuka and colleagues designed a mouse model system that consisted of tail vein injection of B16 melanoma or Lewis lung carcinoma in mice with and without established subcutaneous primary tumors. They reported that MMP-9 expression in pre-metastatic lung endothelial cells and macrophages increased melanoma and lung cancer cell metastasis to the lung [47]. Taken together with reported data that show bone marrow transplant of MMP-9 competent cells into MMP-9 deficient mice enhanced A549 lung cancer cell survival and establishment in the lung emphasize the influence of niches in tumor growth [48].

MMP activity is balanced by TIMP activity. TIMP-2 has been shown to inhibit many MMPs, including MMP-2 (gelatinase A), MMP9 (gelatinase B), and MT1-MMP (MMP-14) and expression of TIMP2 is decreased in invasive and metastatic tumors including prostate, head and neck, lymphoid, and cervical cancers. Overexpressing TIMP2 into murine Lewis lung carcinoma, murine colon carcinoma, and human breast, ovarian, and gastric cancer cells resulted in reduced tumor growth, angiogenesis and metastasis [49,50]. In addition to inhibiting MMPs, TIMP-2 functions to inhibit angiogenesis through an MMP-independent mechanism(s). TIMP-2 can bind $\alpha_{3} \beta_{1}$ integrins on endothelial cells to activate $\mathrm{SH} 2$-containing protein tyrosine phosphatase-1 (SHP-1) that suppresses the activation of Receptor Tyrosine Kinase (RTK) [15]. Additionally, TIMP-2 Loop 6 can bind to the insulin growth factor receptor I (IGF-IR) on endothelial cells thus inhibiting angiogenesis [51]. Overexpression of TIMP-2 or Ala+TIMP-2, a mutant form of TIMP-2 that lacks the ability to inhibit MMPs, reduced A549 lung cancer growth and angiogenesis and increase tumor cell apoptosis [50].

\section{Concluding Remarks}

Advancements in knockout and transgenic mouse technology have generated a wealth of information regarding tumor cell growth, invasion, and dissemination. This has helped to generate many new therapeutic targets for cancer treatment. The interaction of tumor cells with the microenvironment at primary and distant sites is complicated and evolving. As more mechanistic details emerge on the complicated processes of tumor invasion, progression, angiogenesis, and metastasis that involve both tumor and host cell responses a greater the number of potential therapeutic targets will be identified. These new discoveries will result in more specific and personalized therapeutic treatments that can be designed for the individual cancer patient. 
Citation: Moss LAS, Stevenson WGS (2013) Influence of Stromal Components on Lung Cancer Carcinogenesis. J Carcinogene Mutagene S13: 008. doi:10.4172/2157-2518.S13-008

\section{Acknowledgement}

This work was funded by the $\mathrm{NCl}$ Center for Cancer Research Intramural

Research Program Grant \# ZIA SC009179-22.

\section{References}

1. Goldmann E (1908) The Growth of Malignant Disease in Man and the Lowe Animals, with special reference to the Vascular System. Proc R Soc Med 1 1-13.

2. Dvorak HF (1986) Tumors: wounds that do not heal. Similarities between tumor stroma generation and wound healing. N Engl J Med 315: 1650-1659.

3. Folkman J (1971) Tumor angiogenesis: therapeutic implications. N Engl J Med 285: $1182-1186$.

4. Salsbury AJ, Burrage K, Hellmann K (1970) Inhibition of metastatic spread by I.C.R.F. 159: selective deletion of a malignant characteristic. Br Med J 4: 344346.

5. Dvorak HF, Nagy JA, Berse B, Brown LF, Yeo KT, et al. (1992) Vascular permeability factor, fibrin, and the pathogenesis of tumor stroma formation. Ann N Y Acad Sci 667: 101-111.

6. Tammela T, Enholm B, Alitalo K, Paavonen K (2005) The biology of vascular endothelial growth factors. Cardiovasc Res 65: 550-563.

7. Carmeliet P, Jain RK (2000) Angiogenesis in cancer and other diseases. Nature 407: 249-257.

8. Sahni A, Simpson-Haidaris PJ, Sahni SK, Vaday GG, Francis CW (2008) Fibrinogen synthesized by cancer cells augments the proliferative effect of fibroblast growth factor-2 (FGF-2). J Thromb Haemost 6: 176-183.

9. Andreasen PA, Egelund R, Petersen HH (2000) The plasminogen activation system in tumor growth, invasion, and metastasis. Cell Mol Life Sci 57: 25-40.

10. Sivridis E, Giatromanolaki A, Koukourakis MI (2004) "Stromatogenesis" and tumor progression. Int J Surg Pathol 12: 1-9.

11. Giatromanolaki A, Sivridis E, Koukourakis MI (2007) The Pathology of Tumor Stromatogenesis. Cancer Biol Ther 6

12. Bremnes RM, Donnem T, Al-Saad S, Al-Shibli K, Andersen S, et al. (2011) The role of tumor stroma in cancer progression and prognosis: emphasis on carcinoma-associated fibroblasts and non-small cell lung cancer. Journal of thoracic oncology: official publication of the International Association for the Study of Lung Cancer 6: 209-217.

13. Cekanova M, Masi T, Plummer HK 3rd, Majidi M, Fedorocko P, et al. (2006) Pulmonary fibroblasts stimulate the proliferation of cell lines from human lung adenocarcinomas. Anticancer Drugs 17: 771-781.

14. Rucci N, Sanità $P$, Angelucci $A$ (2011) Roles of metalloproteases in metastatic niche. Curr Mol Med 11: 609-622.

15. Stetler-Stevenson WG (2008) Tissue inhibitors of metalloproteinases in cell signaling: metalloproteinase-independent biological activities. Sci Signal 1: re6.

16. Shuman Moss LA, Jensen-Taubman S, Stetler-Stevenson WG (2012) Matrix metalloproteinases: changing roles in tumor progression and metastasis. Am J Pathol 181: 1895-1899.

17. Moore CS, Crocker SJ (2012) An alternate perspective on the roles of TIMPs and MMPs in pathology. Am J Pathol 180: 12-16

18. Schumacker PT, Samsel RW (1989) Analysis of oxygen delivery and uptake relationships in the Krogh tissue model. J Appl Physiol 67: 1234-1244.

19. Hanahan D, Folkman J (1996) Patterns and emerging mechanisms of the angiogenic switch during tumorigenesis. Cell 86: 353-364

20. Hanahan D Weinberg RA (2000). The hallmarks of cancer. Cell 100: 57-70.

21. Hicklin DJ, Ellis LM (2005) Role of the vascular endothelial growth factor pathway in tumor growth and angiogenesis. J Clin Oncol 23: 1011-1027.

22. Carmeliet P, Ferreira V, Breier G, Pollefeyt S, Kieckens L, et al. (1996) Abnormal blood vessel development and lethality in embryos lacking a single VEGF allele. Nature 380: 435-439.

23. Ma YP, Yang Y, Zhang S, Chen X, Zhang N, et al. (2010) Efficient inhibition of lung cancer in murine model by plasmid-encoding VEGF short hairpin RNA in combination with low-dose DDP. J Exp Clin Cancer Res 29: 56.
24. Gerber HP, Ferrara N (2005) Pharmacology and pharmacodynamics of bevacizumab as monotherapy or in combination with cytotoxic therapy in preclinical studies. Cancer Res 65: 671-680.

25. French KJ Kendall SF - 0.

26. Guo J, Higashi K, Ueda Y, Ishigaki Y, Sakuma T, et al. (2011) VEGF-A and its isoform VEGFâ, â,,â, mRNA expression measured by quantitative realtime RT-PCR: correlation with F-18 FDG uptake and aggressiveness of lung adenocarcinoma: preliminary study. Ann Nucl Med 25: 29-36.

27. Yuan A, Lin CY, Chou CH, Shih CM, Chen CY, et al. (2011) Functional and structural characteristics of tumor angiogenesis in lung cancers overexpressing different VEGF isoforms assessed by DCE- and SSCE-MRI. PLoS One 6: e16062.

28. Palumbo JS, Kombrinck KW, Drew AF, Grimes TS, Kiser JH, et al. (2000) Fibrinogen is an important determinant of the metastatic potential of circulating tumor cells. Blood 96: 3302-3309.

29. Palumbo JS, Potter JM, Kaplan LS, Talmage K, Jackson DG, et al. (2002) Spontaneous hematogenous and lymphatic metastasis, but not primary tumor growth or angiogenesis, is diminished in fibrinogen-deficient mice. Cancer Res 62: 6966-6972.

30. Palumbo JS, Talmage KE, Massari JV, La Jeunesse CM, Flick MJ, et al. (2005) Platelets and fibrin(ogen) increase metastatic potential by impeding natura killer cell-mediated elimination of tumor cells. Blood 105: 178-185

31. Malik G, Knowles LM, Dhir R, Xu S, Yang S, et al. (2010) Plasma fibronectin promotes lung metastasis by contributions to fibrin clots and tumor cell invasion. Cancer Res 70: 4327-4334.

32. Sakai T, Johnson KJ, Murozono M, Sakai K, Magnuson MA, et al. (2001) Plasma fibronectin supports neuronal survival and reduces brain injury following transient focal cerebral ischemia but is not essential for skin-wound healing and hemostasis. Nature medicine 7: 324-330.

33. Jia D, Yan M, Wang X, Hao X, Liang L, et al. (2010) Development of a highly metastatic model that reveals a crucial role of fibronectin in lung cancer cel migration and invasion. BMC Cancer 10: 364

34. Bugge TH, Flick MJ, Daugherty CC, Degen JL (1995) Plasminogen deficiency causes severe thrombosis but is compatible with development and reproduction. Genes Dev 9: 794-807.

35. Carmeliet P, Schoonjans L, Kieckens L, Ream B, Degen J, et al. (1994) Physiological consequences of loss of plasminogen activator gene function in mice. Nature 368: 419-424.

36. Cesarman-Maus G, Hajjar KA (2005) Molecular mechanisms of fibrinolysis. Br J Haematol 129: 307-321.

37. Bugge TH, Kombrinck KW, Xiao Q, Holmbäck K, Daugherty CC, et al. (1997) Growth and dissemination of Lewis lung carcinoma in plasminogen-deficient mice. Blood 90: 4522-4531.

38. Casar B, He Y, Iconomou M, Hooper JD, Quigley JP, et al. (2012). Blocking of CDCP1 cleavage in vivo prevents Akt-dependent survival and inhibits metastatic colonization through PARP1-mediated apoptosis of cancer cells. Oncogene 31: 3924-3938.

39. Coussens LM, Fingleton B, Matrisian LM (2002) Matrix metalloproteinase inhibitors and cancer: trials and tribulations. Science 295: 2387-2392.

40. Stetler-Stevenson WG (1996) Dynamics of matrix turnover during pathologic remodeling of the extracellular matrix. Am J Pathol 148: 1345-1350.

41. Yoshida S, Takahashi $\mathrm{H}$ (2009) Expression of extracellular matrix molecules in brain metastasis. J Surg Oncol 100: 65-68.

42. Zeng ZS, Guillem JG (1995) Distinct pattern of matrix metalloproteinase 9 and tissue inhibitor of metalloproteinase 1 mRNA expression in human colorectal cancer and liver metastases. Br J Cancer 72: 575-582.

43. Itoh T, Tanioka M, Yoshida H, Yoshioka T, Nishimoto H, et al. (1998) Reduced angiogenesis and tumor progression in gelatinase A-deficient mice. Cancer Res 58: 1048-1051.

44. Itoh T, Tanioka M, Matsuda H, Nishimoto H, Yoshioka T, et al. (1999) Experimental metastasis is suppressed in MMP-9-deficient mice. Clin Exp Metastasis 17: 177-181.

45. Rao JS, Gondi C, Chetty C, Chittivelu S, Joseph PA, et al. (2005) Inhibition of invasion, angiogenesis, tumor growth, and metastasis by adenovirus-mediated 
Citation: Moss LAS, Stevenson WGS (2013) Influence of Stromal Components on Lung Cancer Carcinogenesis. J Carcinogene Mutagene S13: 008. doi:10.4172/2157-2518.S13-008

Page 5 of 5

transfer of antisense uPAR and MMP-9 in non-small cell lung cancer cells. Mol Cancer Ther 4: 1399-1408.

46. Cao J, Chiarelli C, Kozarekar P, Adler HL (2005) Membrane type 1-matrix metalloproteinase promotes human prostate cancer invasion and metastasis. Thromb Haemost 93: 770-778.

47. Hiratsuka S, Nakamura K, Iwai S, Murakami M, Itoh T, et al. (2002) MMP9 induction by vascular endothelial growth factor receptor-1 is involved in lungspecific metastasis. Cancer Cell 2: 289-300.

48. Acuff HB, Carter KJ, Fingleton B, Gorden DL, Matrisian LM (2006) Matrix metalloproteinase- 9 from bone marrow-derived cells contributes to survival but not growth of tumor cells in the lung microenvironment. Cancer Res 66: 259266.
49. Li H, Lindenmeyer F, Grenet C, Opolon P, Menashi S, et al. (2001) AdTIMP-2 inhibits tumor growth, angiogenesis, and metastasis, and prolongs survival in mice. Hum Gene Ther 12: 515-526.

50. Bourboulia D, Jensen-Taubman S, Rittler MR, Han HY, Chatterjee T, et al. (2011) Endogenous angiogenesis inhibitor blocks tumor growth via direct and indirect effects on tumor microenvironment. Am J Pathol 179: 2589-2600.

51. Fernandez CA, Roy R, Lee S, Yang J, Panigrahy D, et al. (2010) The antiangiogenic peptide, loop 6 , binds insulin-like growth factor-1 receptor. J Bio Chem 285: 41886-41895.
This article was originally published in a special issue, Cell-Extracellular Matrix Interactions in Carcinogenesis handled by Editor(s). Dr. Claudio Luparello, University of Palermo, Italy 